\title{
The multiple vigilance test
}

\author{
MAX HIRSHKOWITZ \\ Veterans Affairs Medical Center and Baylor College of Medicine, Houston, Texas
}

LUPITA DE LA CUEVA

University of Texas Southwestern Medical Center, Dallas, Texas

and

JOHN H. HERMAN

University of Texas Southwestern Medical Center and Children's Medical Center, Dallas, Texas

\begin{abstract}
The multiple vigilance test is a 30 -min signal detection task designed to assess performance. Sixty target and 180 nontarget stimuli are presented with random interstimulus intervals ranging from 4 to $11 \mathrm{sec}$. Subjects respond by pressing the spacebar (or microswitch in an alternate version of the program) when they see the masking pattern transformed into the target. This vigilance test is used in conjunction with measures of physiological sleepiness (Multiple Sleep Latency Test) and subjective sleepiness (Stanford Sleepiness Scale). This behavioral information concerning manifest sleepiness provides a more complete picture of a subject's state of alertness. The assessment of vigilance during long, monotonous, nonstimulating tasks is clinically important in patients with disorders of sleep and arousal.
\end{abstract}

In a report to Congress this year, the National Commission on Sleep Disorders Research estimated that more than 25 million Americans have serious sleep problems. Investigations revealed sleep deprivation as a significant contributor to the Exxon Valdez, Three Mile Island, and Chernobyl disasters. These well-publicized catastrophes overshadow the equally tragic multitude of motor vehicle accidents, many of which are clearly sleepiness related. At normal highway speed (80-90 ft per second), even a 3-sec microsleep episode can produce devastating results. Sleep-related motor vehicle accidents kill more than 10,000 Americans annually and cost society an estimated $\$ 30$ billion.

Clinical sleep disorders centers can now be found in virtually every major city and large medical facility. Clinical sleep specialists customarily assess sleep disorders using nocturnal polysomnography. Standard polysomnography includes recording of electroencephalogram, electrooculogram, electromyogram, respiration, leg movements, heart rhythm, and oximetry. This electrophysiological montage is designed to detect pathophysiologies associated with the most common sleep disorders. Daytime sleepiness is assessed using a polygraphic technique called the Multiple Sleep Latency Test (MSLT; Carskadon et al., 1986). By determining the latency to sleep during four or five nap opportunities scheduled at 2-h intervals throughout the day, sleep tendency can be objectively measured. Sleep tendency is used to index physiological sleepiness. Another aspect of sleepiness, subjective sleepi-

Correspondence should be addressed to M. Hirshkowitz, VAMC Research 116A, 2002 Holcombe Blvd., Houston, TX 77030. ness, is commonly assessed with the Stanford Sleepiness Scale (SSS; Hoddes, Zarcone, Smythe, Phillips, \& Dement, 1973). It is heuristically useful to evaluate introspective and physiological sleepiness in conjunction with one another. What is lacking, however, is a standardized measure of manifest sleepiness.

Measurement of manifest sleepiness has been investigated using a variety of performance tasks. Studies indicate that long, experimenter-paced, monotonous tasks that tax the subject's endurance are sensitive to sleep loss. The consistency of time-on-task effects led Wilkinson (1968) to conclude that such tests should have a duration of at least $30 \mathrm{~min}$. These test characteristics form what is usually called a test of vigilance.

One of the more commonly used vigilance tests is the one developed by Wilkinson (Horne, Anderson, \& Wilkinson, 1983). In it, auditory tones 0.45 and $0.50 \mathrm{sec}$ in duration are presented. The subject attempts to detect the shorter tones during a 30-min test period. After using a Wilkinson-like test during a trial period, we decided to develop the Multiple Vigilance Test (MVT) for several reasons. First, auditory stimuli can produce an alerting response in an inattentive subject. Second, although the tone-length differences are suprathreshold, signal detection in the Wilkinson task was too difficult for some of our cognitive-impaired patients. Finally, we wanted a test requiring the subject's eyes to be open. The reason for an eyes-open requirement was twofold. First, Dinges and Kribbs (1991) report visual reaction time tasks are more sensitive to sleep loss than are auditory tasks. Second, we wanted to avoid facilitating sleep intrusions during the test. A task allowing sleepy patients to close their eyes may inadvertently recruit sleep. 
We established several design goals for the creation of a simple performance task. We chose a vigilance-type test because this kind of test is known to be sensitive to experimentally induced sleep loss. Also, results obtained using simple signal detection tasks are not characteristically confounded by learning effects. Vigilance testing attempts to mimic the tedious, palling situation of watching for blips on a radar screen or for a ship on the horizon. Several design goals were developed, including:

1. To develop an easily administered vigilance test for use in conjunction with the MSLT and the SSS.

2. To use the most common, cost-effective computer system available (that is, an MS-DOS, PC-compatible system).

3. To design a system requiring minimal hardware.

4. To utilize a simple task appropriate for use with both children and adults.

5. To create a test situation that could be used in connection with measures of physiological and introspective sleepiness.

6. To establish a test compatible with concurrent monitoring of brain activity.

In this study, we compare results from vigilance testing with SSS ratings and MSLT indices in patients with obstructive sleep apnea. Obstructive sleep apnea is characterized by episodic cessation of breathing during sleep resulting from airway collapse. It is commonly associated with fragmented sleep, sleep-related blood oxygen desaturations, and excessive daytime sleepiness.

\section{Method}

Twelve patients ( 5 women, 7 men), with a mean age of 48 years, participated. These patients were evaluated clinically for obstructive sleep apnea, and all had significant symptoms of the disorder.

We performed standard clinical nocturnal polysomnography on each patient. The next day, each patient was tested using the MSLT in conjunction with the SSS and the MVT. These tests were administered five times at 2-h intervals. The MSLT is a standard measure of how rapidly an individual falls asleep when given the opportunity. Each MSLT trial provides 20 min for sleep onset to occur while the individual remains in bed. The SSS is a rating scale of introspective sleepiness.

The MVT is a 30 -min test that is given at $2-\mathrm{h}$ intervais just before each nap opportunity of the MSLT. During the test, the subject sits in front of the computer and attempts to detect and discriminate target from nontarget stimuli. The subjects are instructed to press the spacebar, as quickly as possible, when the target appears. Alternate versions of the program utilize a more accurate microswitch interface for acquiring response times. The subject is also monitored using a video camera mounted just above the computer display. The camera output can be superimposed over the image appearing on the computer screen using a video mixer and can be taped using a standard video cassette recorder. Figure 1 schematically illustrates the MVT testing system apparatus.

We instruct subjects to continually watch the computer screen, on which is displayed a masking stimulus (Figure 2B). The masking stimulus transforms into either a target (Figure 2A) or a nontarget (Figure 2C) stimulus for $250 \mathrm{msec}$ and then returns to the masking pattern. This provides control over the duration of stimulus presentation and avoids problems resulting from differences in phosphor decay rate between computer monitors. The recurrence of the masking grid after presentation interrupts the retinal afterimage.

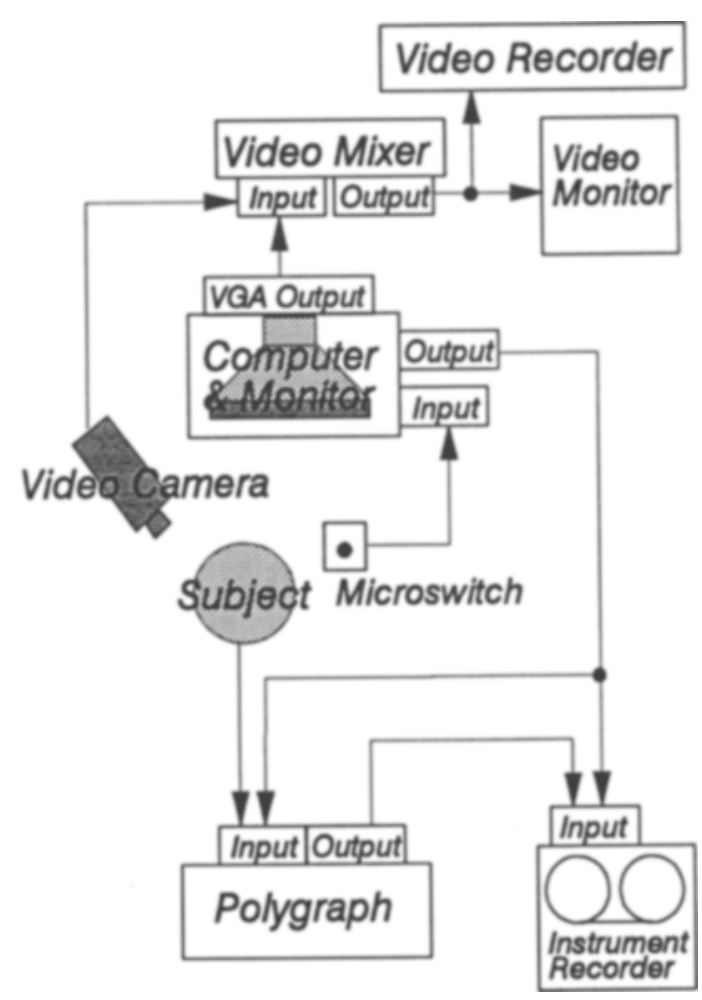

Figure 1. Block diagram of MVT testing apparatus.

The target and nontarget stimuli have an identical number of lines, edges, and contours; however, one is rotated horizontally $90^{\circ}$. The design of stimuli to be identical in all aspects except orientation was to control retinal and cortical stimulation. This controls stimulus properties for concurrent studies of electroencephalographic activity and event-related potentials (Herman et al., 1992).

In total, 60 target and 180 nontarget stimuli are presented. The sequence is random within each 1-min block, with the requirement that each minute contains two targets and six nontargets. A random interstimulus interval, ranging from 4 to $11 \mathrm{sec}$, is used to minimize temporal expectancy. After the onset of each stimulus, the subject has $2 \mathrm{sec}$ in which to respond. Response time for each trial is recorded. Data are stored for each presentation with a sequence number, elapsed time from start of test, stimulus type, and the subject's response time in a user-identified file. Data are tabulated separately for hits, correct rejections, false alarms, and misses.

The MVT system includes programs to administer the test, enter MSLT and SSS data, and summarize results. The administration program has several selectable features. It contains an instruction

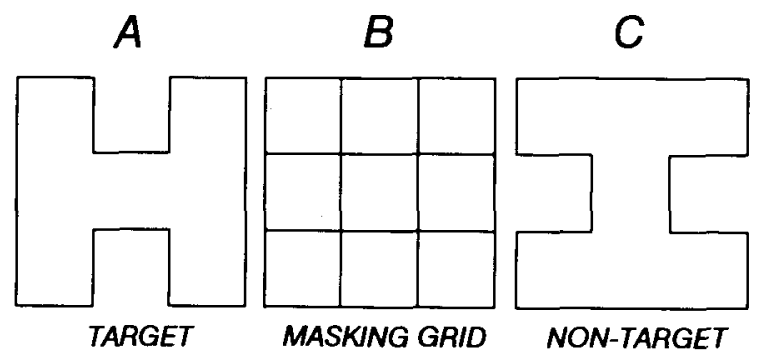

Figure 2. Target, masking grid, and nontarget stimuli presented during vigilance testing. 
screen, an interactive demonstration routine to display target and nontarget stimuli (used to orient the subject), and a practice trial run. The MSLT data entry program creates a report and data file of MSLT results. MVT summary reports can be generated with or without MSLT data. All programs were developed using Microsoft QuickBASIC.

\section{Results}

MSLT tracings were scored for sleep onset using standard criteria (Carskadon et al., 1986). Mean sleep latencies, SSS ratings, MVT response times, and MVT misses were tabulated and analyzed with SAS (SAS Institute, Cary, NC). Mean within-subject response times ranged from 602 to $1,041 \mathrm{msec}$. The within-subject mean number of misses ranged from 3.4 to 25.8 of the 60 possible target stimuli presented per testing session. Participants described their sleepiness ranging from hardly at all to severe on SSS (range: from 2, functioning at high level, but not at the peak; able to concentrate, to 6, sleepiness; would prefer to be lying down; fighting sleep; woozy). Within-subject mean sleep latency ranged from 1 to $13 \mathrm{~min}$.

Scatterplots and Pearson correlations between MVT measures (response times and number of misses) and SSS and MSLT measures are illustrated in Figure 3. Surprisingly, the strongest association was an inverse relationship between MVT response time and SSS rating $(r=-0.63$, $p=.03$ ). We found marginal negative correlations between the number of missed targets and SSS ratings $(r=-0.55$, $p=.07)$ and MSLT sleep tendency $(r=-0.50, p=$ $.10)$. By contrast, response time and mean sleep latency were unrelated $(r=-0.19, p=.55)$.

Figure 4 illustrates electroencephalographic, electrooculographic, electromyographic, and electrocardiographic
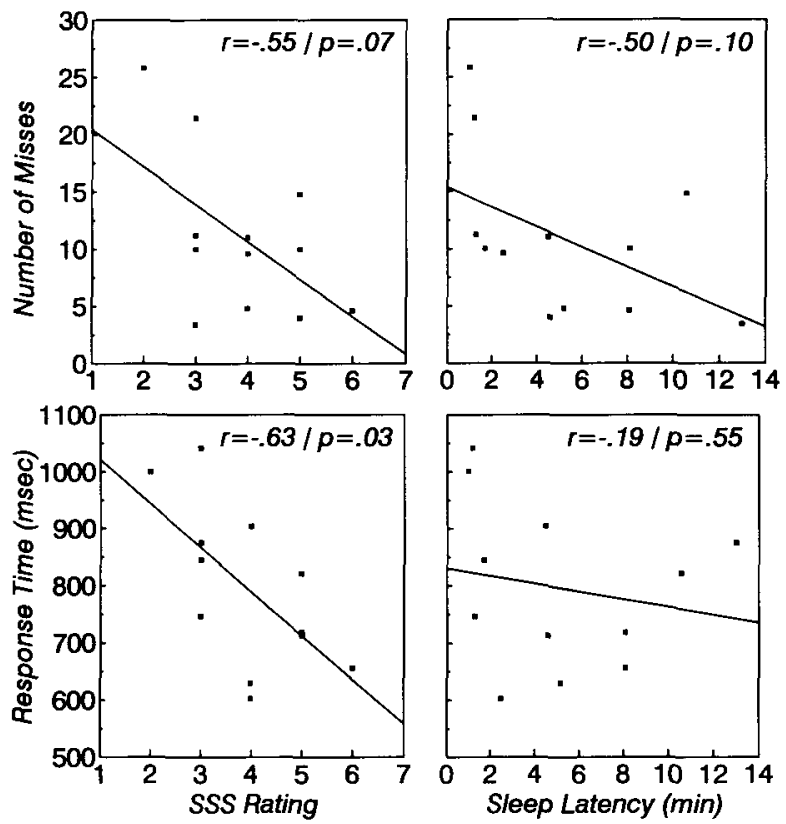

Figure 3. Scatterplots with regression lines for introspective sleepiness (SSS rating) and physiological sleepiness (MSLT sleep latency) against MVT measures (number of misses and response times).

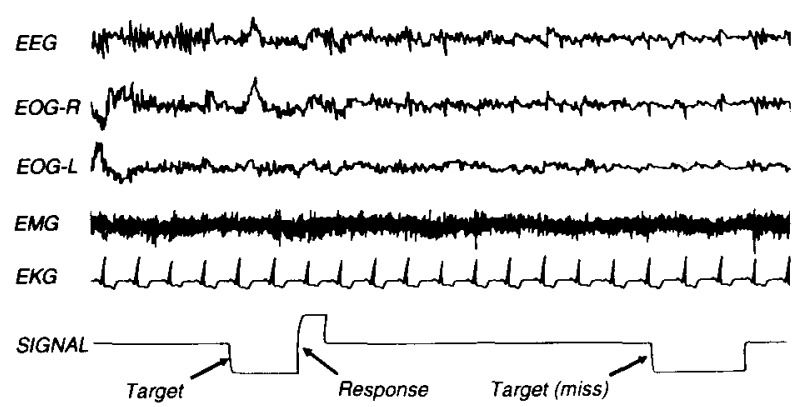

Figure 4. Electrophysiological concomitants of failure to respond to a target stimulus. The evolution of sleep is observable as alpha EEG activity disappears just before the missed target stimulus is presented. The patient's eyes were open and direction of gaze was toward the stimulus presentation field.

activity during MVT testing. Brain activity and video monitoring substantiate the presence of eyes-open microsleep during some target misses.

\section{Discussion}

The results of this study indicate that the MVT provides additional information useful in evaluating disorders of excessive daytime sleepiness. The behavioral measure of performance compliments introspective and physiological measures; however, it does not duplicate them. Our results indicate a paradoxical association between these patients' subjective estimates of sleepiness and their performance level. Individuals self-reporting greater alertness performed more poorly than did those indicating sleepiness. These data emphasize the unreliability of selfreported sleepiness in impaired individuals and provide insight into a person's inability to predict and thereby avoid sleep-related accidents. The negative relationship between measures of introspective and manifest sleepiness may stem from mental distortions produced by or adaptation to a condition of chronic sleep disturbance.

By contrast, a modest association was found between physiological and manifest sleepiness. Response lapses were common in those patients who fell asleep rapidly when given the opportunity. The use of video monitoring, overlaid with an image of the actual test screen, provides the facility to review each response lapse. This feature allows us to differentiate eyes-open attentive microsleep from eyes-closed misses, inattentiveness, or distraction. Using MVT in conjunction with brain activity monitoring helps control some of the confounding factors in previous studies of microsleep.

Each 30-min MVT trial is administered just before MSLT nap opportunities at 2-h intervals. Scheduling testing before, rather than after, nap attempts avoids performance modification by actual sleep occurring during the MSLT trial. Many subjects find naps restorative. By contrast, napping produces postsleep inertia or a "sleep hangover" in some individuals. The structured nature of the MVT testing also helps standardize the subject's routine leading up to each nap attempt. In accordance with stan- 
dard MSLT procedure, we record a nocturnal polysomnogram the night before testing.

During each MVT trial, 60 target and 180 nontarget stimuli are presented. Horne et al. (1983) indicate that a 1:3 target:nontarget presentation ratio is sensitive to sleep loss. We find that, notwithstanding the fairly high rate of stimulus presentation for a watch-keeping task, the 4-11-sec random interstimulus interval helps offset temporal expectancy. Our experimental subjects and clinical patients have spontaneously remarked that this test is certainly tedious and boring.

We are currently using the MVT to study adult and pediatric patients at our sleep disorders centers (Herman et al., 1990; Herman et al., 1992). In these studies, we compare patients with excessive daytime sleepiness, impaired attentiveness, or both with age-matched control subjects. We are also evaluating the effect of sleep loss on MVT performance. Finally, using an optional digitalto-analog converter, we can record an encoded signal designating stimulus presentation, stimulus type, and response latency onto magnetic tape. This marker, when recorded with concurrent electroencephalographic activity, can be used to examine physiological changes associated with loss of vigilance. These data are useful for exploring both microsleep episodes and visual eventrelated potential alterations associated with changes in levels of alertness.

\section{Availability of Programs}

All programs and documentation needed to utilize the MVT are available from the authors upon request.

\section{REFERENCES}

Carskadon, M. A., Dement, W. C., Mitler, M. M., Roth, T., Westbrook, P. R., KEENAN, S. (1986). Guidelines for the multiple sleep latency test (MSLT): A standard measure of sleepiness. Sleep, 9, 519-524.

Dinges, D. F., KRIBBS, N. B. (1991). Performing while sleepy: Effects of experimentally-induced sleepiness. In T. H. Monk (Ed.), Sleep, sleepiness and performance (pp. 98-128). Chinchester: Wiley.

Herman, J., lalitani, K., Polakoff, R., armitage, R., RoffWARG, H., Hirshkowitz, M. (1992). Human vigilance and the visual evoked potential. Sleep Research, 21, 24.

Herman, J. H., Steinberg, J. B., Roffwarg, R. P., Becker, P. M., Jamieson, A. O., Brown, W. D., Davis, W. P., Fox, N. T., Polakoff, R. J., \& Hirshkowitz, M. (1990). Vigilance and sleepiness in the hypersomnolent child. Sleep Research, 19, 119.

Hoddes, E., Zarcone, V., Smythe, H., Phillips, R., Dement, W. C. (1973). Quantification of sleepiness: A new approach. Psychophysiology, 10, 431-436.

Horne, J. A., ANDERSon, N. R., \& Wilkinson, R. T. (1983). Effects of sleep deprivation on signal detection measures of vigilance: Implications for sleep function. Sleep, 6, 347-358.

WILKINson, R. T. (1968). Sleep deprivation: Performance tests for partial and selective sleep deprivation. In L. E. Abt \& B. F. Riess (Eds.), Progress in clinical psychology: Dreams and dreaming (pp. 28-43). New York: Grune and Stratton. 